\title{
Retroperitoneal hematoma following radical orchiectomy: Two cases
}

\author{
Rachel Glicksman, MD;1 Robert J. Hamilton, MD,2,3 Peter Chung, MD ${ }^{1,4}$
}

'Department of Radiation Oncology, University of Toronto, Toronto, ON, Canada; ${ }^{2}$ Department of Surgery (Urology), University of Toronto, Toronto, ON, Canada; ${ }^{3}$ Department of Surgery (Urology), Princess Margaret Cancer Centre, Toronto, ON, Canada; ${ }^{4}$ Radiation Medicine Program, Princess Margaret Cancer Centre, Toronto, ON, Canada

Cite as: Can Urol Assoc J 2017;11 (1-2):E35-7. http://dx.doi.org/10.5489/cuaj.3996

Published online January 12, 2017

\section{Abstract}

Treatment of testicular cancer is dependent on the stage of disease at presentation. Stage 1 testicular cancer is treated with radical orchiectomy, followed by active surveillance, radiotherapy, or chemotherapy. Occasionally, unusual and unexpected postoperative changes can be seen on computed tomography $(\mathrm{CT})$, and may raise concern for metastatic disease.

Here, we present two cases of testicular cancer patients who developed retroperitoneal hematomas post-radical orchiectomy, one as a classical clinical presentation, and the other as an atypical radiological entity only. The first is a case of a 38-year-old male with a non-seminoma testicular cancer, who developed severe flank pain, hemodynamic instability, and progressive anemia from a retroperitoneal hematoma in the immediate $(<24$ hours) postoperative period, requiring urgent surgical evacuation. The second is a case of a 33-year-old male with a testicular seminoma who had a large, suspicious retroperitoneal mass on a staging CT scan concerning for metastatic disease, which was later diagnosed as a retroperitoneal hematoma. These cases reveal the clinical variability with which a retroperitoneal hematoma post-radical orchiectomy may present. In addition, the second case demonstrates the importance of recognizing radiological postoperative changes and ensuring that these findings are not mistaken for and treated as metastatic disease.

\section{Introduction}

Testicular cancer is the most common malignancy in men aged $15-34^{1}$ years and treatment is dependent on the stage of disease at presentation. Abdominal and pelvic computed tomography (CT) is a well-established component of staging to assess for lymph node involvement and distant metastases. ${ }^{2}$ In men with stage 1 testicular cancer, standard treatment is radical inguinal orchiectomy followed by active surveillance, radiotherapy, or chemotherapy. ${ }^{3-5}$ Although the vast majority of imaging done as part of staging investigations following radical orchiectomy accurately identifies disease, occasionally post-surgical changes may confuse the picture.

We present two cases of post-radical orchiectomy retroperitoneal hematomas (RPH). The first case represents a more classic clinical presentation. The second case is atypical, as the hematoma was found incidentally on CT.

\section{Case report 1}

A 38-year-old male presented with right scrotal swelling, confirmed on ultrasound. Bloodwork revealed lactate dehydrogenase $(\mathrm{LDH}) 322 \mathrm{U} / \mathrm{L}$, human chorionic gonadotropin (hCG) $5558 \mathrm{IU} / \mathrm{L}$, and alpha-fetoprotein (AFP) $1830 \mathrm{ug} / \mathrm{L}$ (all significantly elevated). Radical inguinal orchiectomy revealed a mixed germ cell tumour.

Surgery was complicated by spermatic cord bleeding, however, apparent hemostasis was achieved intraoperatively. Twelve hours postoperatively, the patient developed right inguinal pain, vomiting, and hemoglobin of $74 \mathrm{~g} / \mathrm{L}$. The patient was taken to the operating room for emergent wound exploration, where a RPH was identified with active bleeding from the cord. Hemostasis was obtained and the hematoma was evacuated. His subsequent postoperative course was uncomplicated and he was discharged home three days later.

He was placed on active surveillance postoperatively and at three months after his orchiectomy, imaging revealed an enlarged aortocaval lymph node, with elevated tumour markers (HCG $85 \mathrm{IU} / \mathrm{L}$, AFP $11 \mathrm{ug} / \mathrm{L}$ ). He was treated with chemotherapy and had a complete response.

\section{Case report 2}

A 33-year-old male presented with a palpable right scrotal mass, confirmed on ultrasound. Radical inguinal orchiectomy revealed a unifocal $4.3 \mathrm{~cm}$ seminoma. Tumour markers were normal. Preoperative CT abdomen/pelvis revealed a non-specific right common iliac node. He was categorized as stage 1 seminoma and placed on an active surveillance protocol. 
CT abdomen/pelvis performed three months postoperatively revealed a hypodense lesion with an enhancing rim in the right abdomen (Fig. 1). Initially this was thought to represent recurrent adenopathy with necrosis. The patient underwent discussions regarding the definitive treatment for relapse (chemotherapy). However, further imaging review prior to the start of treatment brought forward additional differential diagnoses of hematoma, seroma, and abscess. The patient was clinically stable with no bloodwork abnormalities. Ultrasound confirmed an avascular tubular cystic lesion right to the midline and anterior to the IVC measuring $13.5 \mathrm{~cm}$ by $5.5 \mathrm{~cm}$ by $4.2 \mathrm{~cm}$. Given the uncertainty of this representing a relapse, it was elected to repeat the ultrasound one month later. This revealed a decrease in the size of the lesion, thus favouring a hematoma. Serial imaging 15 months postoperatively revealed slow interval decrease in the size of the retroperitoneal mass.

At one-year postoperatively, two enlarged right common iliac lymph nodes were identified on CT. Ultrasound-guided biopsy revealed metastatic seminoma. The patient underwent salvage radiotherapy, 35 Gy in 20 fractions to his abdomen/pelvis. At his three-month followup, he had complete resolution of his disease.

\section{Discussion}

RPHs in the post-orchiectomy setting are a rare occurrence. There are older case reports discussing acute and insidious presentations of RPHs, similar to the cases we described above. ${ }^{6-11}$ The mechanism by which this phenomenon occurs can be understood by considering the surgical technique of radical orchiectomy. The spermatic cord is identified, mobilized, and isolated. The vas deferens is ligated and divided with a suture. The remainder of the spermatic cord containing the testicular artery and vein is ligated and divided at the internal abdominal ring. ${ }^{7-9}$ If the divided vessel within the ligature does not reach optimal hemostasis, or retracts or slips beneath the suture, the vessel could withdraw into the canal, resulting in a hemorrhage tracking along the course of the vessels into the retroperitoneum, giving the appearance of a retroperitoneal mass. ${ }^{7-9}$

Hematomas can look similar to metastatic disease on conventional CT imaging. ${ }^{6-10}$ They commonly appear as a mass, compressing normal surrounding structures. ${ }^{8}$ Initially, the density of a hematoma is heterogeneous, with some predominant areas of hyperdensity compared to soft tissue, often in the centre of the mass. ${ }^{7,9,10}$ With time, the hematoma evolves and the attenuation values become isodense and then hypodense with respect to soft tissue. ${ }^{9}$ Hematomas should be kept in the differential diagnosis of these lesions, particularly if specific radiographical features associated with metastases are not seen. Ultimately, further investiga-

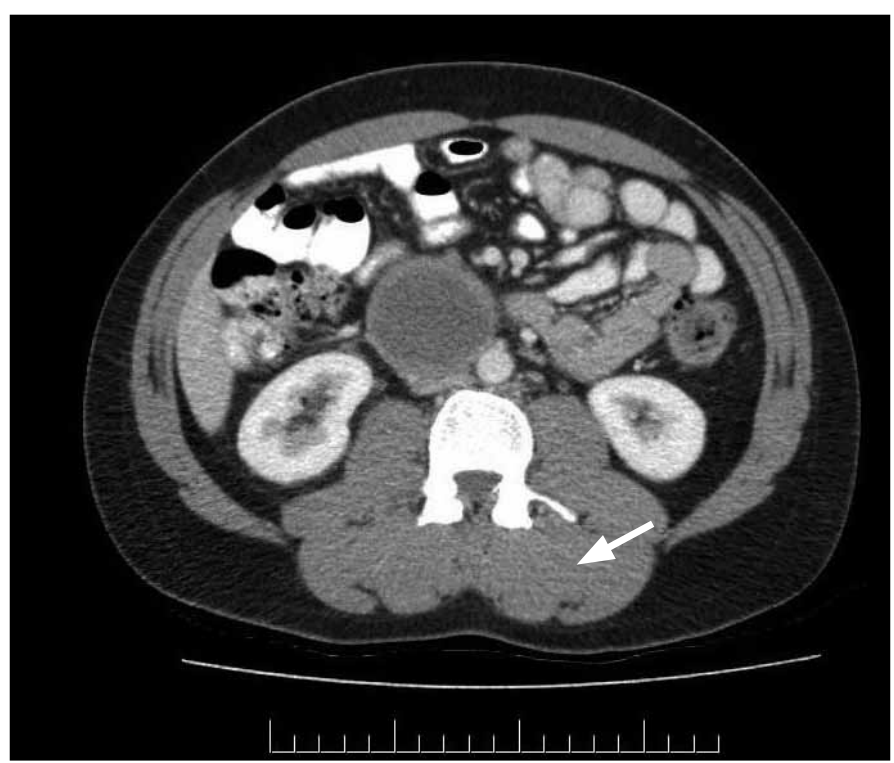

Fig. 1. Three-month postoperative abdominal/pelvic computed tomography axial slice. There is a hypodense tubular rim-enhancing lesion measuring 5.3 $\mathrm{cm} \times 5.7 \mathrm{~cm}$ in the right abdomen.

tions with magnetic resonance (MR) or biopsy of the suspicious lesion may be necessary to exclude metastatic disease.

The second case presented as an unexpected radiological finding and was interpreted as metastatic disease, initiating discussion about treatment. Surveillance as a management strategy in stage 1 disease is widely adopted as the preferred choice for many patients. ${ }^{3,4}$ Serial imaging is performed to detect relapses, which most frequently occur in the retroperitoneum. The uncommon occurrence of subclinical RPHs on surveillance imaging may complicate the otherwise usual course of followup of these patients. This finding in our second patient created a complicated clinical and radiological picture. When his relapse was detected, it was difficult to discern, given that one mass (hematoma) was reducing in size, while the other (metastasis) vas growing on his surveillance scans. Although this is unlikely to impact the patient's outcome, as cure rates approach $100 \%$ with salvage therapies, there are known morbidities to salvage treatment, ${ }^{1,3,4,12,13}$ and it was important that he was not treated based on the complicated imaging alone, but rather when metastatic disease was confirmed.

We recently examined whether there is an association with the institution where orchiectomies are performed (i.e., high-volume academic vs. community centres), and outcomes of active surveillance for stage 1 disease. ${ }^{14}$ We observed no difference in outcomes, suggesting that either the adverse event rate (such as hematoma) is sufficiently low that no difference could be detected or, more likely, that orchiectomy may be safely performed at any institution. However, other aspects of testicular cancer care, particu- 
larly in those with advanced disease should be managed at experienced centres. ${ }^{15}$

Competing interests: Dr. Glicksman has received a research grant from AbbVie. Dr. Hamilton has been an advisor for AbbVie, Astellas, Bayer, and Janssen; and has participated in clinical trials for Janssen. Dr. Chung has received honoraria from AbbVie and Sanofi, and has participated in a clinical trial for AbbVie.

This paper has been peer-reviewed.

\section{References}

1. Groll RJ, Warde P, Jewett MA. A comprehensive systematic review of testicular germ cell tumour surveillance. Crit Rev Oncol Hematol 2007;64:182-97. https://doi.org/10.1016/i.critrevonc.2007.04.014

2. Network NCC. NCCN Clinical Practice Guidelines in Oncology for Testicular Cancer. 2012.

3. Chung P, Warde P. Testicular cancer: Seminoma. BMJ Clin Evid 2011:2011.

4. Kollmannsberger C, Tandstad T, Bedard PL, et al. Patterns of relapse in patients with clinical stage 1 testicular cancer managed with active surveillance. J Clin Oncol 2015;33:51-7. https://doi.org/10.1200/ JC0.2014.56.2116

5. Sturgeon JF, Moore MJ, Kakiashvili DM, et al. Non-risk-adapted surveillance in clinical stage 1 nonseminomatous germ cell tumours: The Princess Margaret Hospital's experience. Eur Urol 201 1:59:556-62. https://doi.org/10.1016/i.eururo.2010.12.010

6. Bochner BH, Lerner SP, Kawachi M, et al. Post-radical orchiectomy hemorrhage: Should an alteration in staging strategy for testicular cancer be considered? Urology 1995;46:408-11. https://doi.org/10.1016/ S0090-4295(99)80232-5
7. Kullmann G, Lien HH. Intraabdominal hematoma following orchiectomy: A potential piffall in using CT for staging of testicular cancer. Radiology 1987;163:129-30. https://doi.org/10.1148/radiology.163.1.3823424

8. Page JE, Prendergast CM, King DM. Retroperitoneal hematoma following orchidectomy: Implications for staging computed tomography. Br J Radiol 1990;63:490-2. https://doi.org/10.1259/0007-128563-750-490

9. Russell SA, Johnson RJ, Russell JM. Pelvic hematoma following orchidectomy: A piffall in the staging of non-seminomatous germ cell tumour. Br J Radiol 1990;63:492-4. https://doi.org/10.1259/00071285-63-750-492

10. Tran T, Grech P, Crofton ME. Computed tomographic staging of testicular tumours: An unexpected source of error. Br J Radiology 1989;62:942-4. https://doi.org/10.1259/0007-1285-62-742-942

11. Kennedy C, Harland C, Watson G, et al. Retroperitoneal hematoma and pelvic hematoma following orchidectomy. Br J Radiol 1991;64:72-3. https://doi.org/10.1259/0007-1285-64-757-72-b

12. Chung P, Warde P. Contemporary management of stage 1 and 2 seminoma. Curr Urol Rep 2013;14:52533. https://doi.org/10.1007/s11934-013-0365-2

13. Leung $E$, Warde $P$, Jewett $M$, et al. Treatment burden in stage 1 seminoma: $A$ comparison of surveillance and adjuvant radiation therapy. BJU Int 2013;112:1088-95. https://doi.org/10.1111/bju.12330

14. Nayan $M$, Jewett MA, Anson-Cartwright $L$, et al. The association between institution at orchiectomy and outcomes on active surveillance for clinical stage 1 germ cell tumours. Can Urol Assoc J 2016;10:204-9. https://doi.org/10.5489/cuai.3513

15. Krege S, Beyer J, Souchon R, et al. European consensus conference on diagnosis and treatment of germ cell cancer: A report of the second meeting of the European Germ Cell Cancer Consensus group (EGCCCG): part l. Eur Urol 2008;53:478-96. https://doi.org/10.1016/i.eururo.2007.12.024

Correspondence: Dr. Rachel Glicksman, Department of Radiation Oncology, University of Toronto, Toronto, 0N, Canada; rachelmglicksman@gmail.com 